\title{
UN TESTO LATINO-PERSIANO CONNESSO AL CODEX CUMANICUS
}

\author{
ANGElo MiCHELE PiEMONTESE* \\ (Roma)
}

\section{Index}

Il codice lat. DXLIX (1597) della Biblioteca Marciana di Venezia, celebre come "Codex Cumanicus", reca il lessico persicum et Comanicum per alfabetum (PCA). Esso fu dedicato (A. D. 1303: 1330?) «Ad honorem dei et Beati Iohannis euangeliste» (intestazione, f. $\left.1^{\mathrm{r}}, 1.4\right)$. Un frasario tedesco-turco cumano fu aggiunto a $P C A$ in età incerta. Una copia del codice fu eseguita da un anonimo amanuense su committenza di J. H. von Klaproth (c. 1824), primo editore del "Codex Cumanicus". Tale apografo, ms. Ashburnham 1584 della Biblioteca Medicea Laurenziana di Firenze, è rilevante per il confronto paleografico e l'integrazione della lettura dell'antigrafo. ${ }^{1}$

Il "Codex Cumanicus", precisamente il suo tomo I, $P C A$, fu trovato in un gruppo di 17 libri dentro una stanza murata vicina ai Cavalli bronzei, sopra la facciata della basilica di San Marco in Venezia (13.XII.1634). F. Olmi O. S. B., abate cassinese della chiesa veneziana di S. Giorgio Maggiore, esperto di manoscritti e storico (Venezia, c. 1570-1648), compì una ricognizione dei codici trovati, per incarico del Senato della Repubblica veneziana. Olmi scrisse una Relazione al riguardo (1640). Egli vi accluse l'Indice di tali libri, ritenuti il gruppo di codici che il grande letterato umanista Francesco Petrarca (Incisa 1304-Arquà 1374) aveva lasciato in dono a «San Marco Evangelista» (1362), ma poi fu disperso. Olmi trasmise l'Indice a I. F. Tomasini, petrarchista. ${ }^{2}$ Egli pubblicò la lista dei «Codici qui adhuc extant è Bibliotheca

${ }^{*}$ A. M. Piemontese, Dipartimento di Studi Orientali, Univ. di Roma «La Sapienza», P.le Aldo Moro, 5, 00185 Roma.

${ }^{1}$ A. M. Piemontese: Catalogo dei manoscritti persiani conservati nelle biblioteche d'Italia. Roma 1989, pp. 100-101, 342-343. A. Gallotta: Una «nuova» copia del Codex Cumanicus. Annali dell'Istituto Universitario Orientale di Napoli 52 (1992), pp. 455-459.

${ }^{2}$ E. A. Cicogna: Delle iscrizioni veneziane raccolte ed illustrate, IV. Venezia, 1834, pp. 335-340. Cf. M. Armellini: Bibliotheca Benedectino Casinensis sive scriptorum Casinensi Congregationis alias S. Justinae Patavinae Qui in ea ad haec usque tempora floruerunt Operum, ac Gestarum notitiae, I. Assisii, 1731, pp. 173-174. 
Francisci Petrarchae, vt mihi comunicauit D. Fortunatus Vlmus Abbas Cassinensis: Alphabetum Persicum, Comanicum \& Latinum Anonymi scriptum Anno MCIII. Die XI. Iulij. cuius librum initium est tale. In nomine Domini Iesu Christi \&c. Haec sunt prima verba \& nomina de litera A Audio Mesnoem Esiturmen Audis Mesnoy Esitursen Audit Mesnoet Esitin» etc. ${ }^{3}$

La brevissima prima catalogazione del codice riferisce la consistenza e la foliazione, che sono diverse dalle attuali: «Codex DXLIX in 4. chartaceus, foliorum 62. saeculi XIV. LEXICON latinum, Persicum et Comanicum. Initio legitur: MCCCIII. die XI. Iulii.» ${ }^{4}$ L'autenticità della donazione di Petrarca fu ribadita. ${ }^{5}$ I duri termini di una smentita sgomentano ogni codicologo: «un lot de manuscrits détériorés, pour la pluspart postérieurs à Pétrarque, et dont aucun ne peut vener de lui [...: j] jai pu, en 1889, faire disparaître de la Marcienne les restes de l'erreur traditionnelle; on y montrait encore avec une inscription attribuée à Pétrarque le fameux Vocabolario Comano [...]; il n'y a pas une seule trace de la provenance de Pétrarque dans la collection». 6 Il problema della donazione "continuò a rimanere insoluto», a causa della dispersione postuma della libreria personale di Petrarca. ${ }^{7}$ Ma una possibile via di trasmissione di PCA a Petrarca esiste. Il tramite concerne il suo caro amico Giovanni Colonna O. P., erudito e storico.

\section{Coniunctio}

«Iohannes de Columna (de Gallicano) Romanus. Natus a. 1298, nepos Landulphi de Columna. Ante annum 1320, quo studii causa Parisiis morabatur, Ord. Praed. ingressus est. Praedicator gen. (1324). Cappellanus Ioannis de Comitibus de Polo, archiep. Nicosiensis (1312-1332). Avenione in curia pontificia. Vicarius conv. S. Sabinae de Urbe (1338). Lector in conv. Tiburtino (1339). Obiit exeunte anno 1343. Amicitiae vinculo et epistularum commercio cum Francisco Petrarca coniunctus.»»

Riguardo ai viaggi di Colonna (c. 1332-1336) in Persia, Arabia, Egitto (quindi in Terra Santa: Palestina, Siria), Caucaso (quindi Georgia e Armenia), Petrarca fornisce l'unica fonte d'informazione tuttora disponibile. Egli scriveva a Colonna nelle Epistolae Familiares VI.3 (c. 1342): «Agnosce pastoris tui providentiam: non te in Perside, non in Arabia vel Egipto, ubi velut in suburbano vagabaris tuo, sed in patri-

${ }^{3}$ I. Ph. Tomasini: Petrarcha redivivus. Patavii 1650, pp. 56, 70. Id.: Bibliothecae Venetae manuscripta publicae \& privatae, Utini 1650, p. 56.

${ }^{4}$ Latina et italica D. Marci Bibliotheca codicvm manvscriptorvm per titvlos digesta, [A. M. Zanetti ed., Venetiis] 1741, p. 216.

${ }^{5}$ J. Valentinelli: Bibliotheca Manuscripta ad S. Marci Venetiarum. Codices MSS. Latini Tom. I, Venetiis 1868, pp. 2-10. Cf. A. Zardo: Il Petrarca e i Carraresi. Milano 1887, pp. 247$248,316$.

${ }^{6}$ Pierre de Nolhac: Pétrarque et l'humanisme d'après un essai de restitution de sa bibliothèque. Paris 1892 , pp. 83-84, nota 1.

${ }^{7}$ M. Luxoro: La biblioteca di S. Marco nella sua storia. Firenze 1954, pp. 1-11.

${ }^{8}$ Th. Kaeppeli O. P.: Scriptores Ordinis Praedicatorum in Medii Aevi, II. Romae ad S. Sabinae, 1975, pp. 399-400. 
am, integro membrorum obsequio, reversum post peregrinationes innumeras» (§ 59). «Esse nunc, ut auguror, in aliquomundi angulo: iam Nilus, iam Indum aut Tanaim transnatares, iam Ripheos montes aut Hercynii saltus dumeta transcenderes, vagus semper et profugus super terram» (§ 62). E ( $\$ 64)$ «Pone me in supremo Atlantis meduseis luminibus durato vertice, te in rupe Caucasea, ubi vinctus Prometheus de Iove conqueritur; simul sedebimus, simul ambulabimus, simul cenabimus, simul fabulamibur, simul seria tractabimus. ${ }^{9}$

«While working in the papal curia, Colonna wrote a De viribus illustribus; it survives in three manuscripts. Later at Rome he compiled a universal of the world entitled Mare Historiarum» parimenti inedito. ${ }^{10}$ In Cipro Colonna fu cappellano di Giovanni Conti de Polo romano O. P., arcivescovo latino di Nicosia (1324-1332). Prima egli era stato (1299-1312) vescovo di Pisa. ${ }^{11}$ Conti «In illis partibus multos captivos Christianos de manibus saracenorum redemit». Intanto Colonna compiva interviste e compulsava archivi, da cui egli trasse materia per il Mare Historiarum. «John Colonna speaking of the capture of the city of Akka by the Christians in 1191 says that among the Saracen prisoners there was Karakush, the wise minister of Saladin»: Colonna, Mare Historiarum, codex parisinus lat. 4914 (f. 299r), riferisce: «ut in quadam cronica in arabico scripta, quam frater Alphonsus [Buenhombre O. P.] Hispanus transtulit in latinum, repperi.»» ${ }^{12}$

In Chartres, Colonna aveva intervistato il «subcantor in ecclesia cartonensi, Robertus nomine» che era stato compagno di André de Longjumeau O. P., nunzio di Luigi IX re di Francia ad Tartaros. André fu latore della celebre lettera inviata al re di Francia navigante verso Cipro (1248) da Erchaltay, governatore mongolo di Persia. Il re «litteras scriptas in persica lingua primo in graecum, deumm in latinum transferri mandavit», riferisce Colonna. ${ }^{13}$ André de Longjumeau fu il primo frate domenicano europeo buon conoscitore del persiano parlato e scritto. ${ }^{14}$ L'Ordine dei Predicatori curava lo studio di lingue asiatiche per attuare l'evangelizzazione. ${ }^{15}$

${ }^{9}$ F. Petrarca: Le Familiari, ed. V. Rossi. Firenze 1934, II, pp. 74-75. Cf. B. Ross: New Autographs of Fra Giovanni Colonna. Studi Petrarcheschi n. s. II (1985), pp. 211-229. $533-563$.

${ }^{10}$ W. B. Ross: Giovanni Colonna, Historian at Avignon. Speculum XLV (1970), pp.

${ }^{11}$ N. Zucchelli: Cronotassi dei vescovi e arcivescovi di Pisa. Pisa 1907, pp. 109-114; Mas Latrie: Histoire des Archevêques latins de l'île de Chypre. Archives de l'Orient Latin II (1884), pp. 255-261.

${ }^{12}$ S. L. Forte O. P.: John Colonna O. P. Life and Writings (1298 c.-1340). Archivum Fratrum Praedicatorum XX (1950), pp. 369-414.

${ }^{13}$ G. Colonna: Mare Historiarum, in Recueil des Historiens de Gaules et de la France, t. XIII, publié par De Wailly, Delisle et Jourdain. Paris 1876, pp. 106-124: 117.

${ }^{14}$ B. Altaner: Die Dominikanermissionen des 13. Jahrhunderts. Hebelschwerdt 1924, pp. 52-58, 128-130; Paul Pelliot: Les Mongols et la papauté. Revue de l'Orient Chrétien XXIV (1924), pp. 225-335: 288; XXVIII (1931-1932), pp. 3-84.

${ }^{5}$ B. Altaner: Sprachstudien und Sprachkenntnisse im Dienste der Mission des 13. und 14. Jahrhunderts. Zeitschrift für Missionwissenschaft 21 (1931), pp. 113-136; Id.: Die fremdsprachliche Ausbildung der Dominikermissionare während des 13. und 14. Jahrhunderts. ibid., 23 (1933), pp. 233-241; N. Simonut: Il metodo d'evangelizzazione dei Francescani tra musulmani e mongoli nei secoli XIII e XIV. Milano 1947, pp. 30-34, 48-49. 
Nella curia pontificia di Avignone esisteva una scuola orientalistica, dove operavano anche traduttori armeni, fra cui Nersês detto Palianenç. Egli, dal 1338 in Italia, quindi in Avignone, lettore nella curia pontificia, era stato vescovo di Urmia e legato ai Frati Peregrinanti e ai connessi Unitori del monastero di Kirnë, in provincia di Nakhicevan. ${ }^{16}$ Per quanto concerne il conflitto fra Europa e «Gens Turcorum sive Thorcomannorum», inoltre i Tartari, ossia Mongoli, Colonna poteva compulsare anche il Chronicon (anni 754-1314) di Francesco Pipini da Bologna O. P. ${ }^{17}$

Pipini seguiva l'attività che compiva «nell'ordine domenicano la "Società dei frati peregrinanti", una congregazione missionaria per l'evangelizzazione dell'Oriente..${ }^{18}$ Colonna, trattando "De modo quem tenent Tartari in re militari $[. .$.$] et de cer-$ tis aliis eorum moribus", dichiara: «Set et christiani, cuiusque secte sunt, publice et ad partem fidem eorum predicare permittunt, eciam volentes baptismum et fidem recipere non prohibent. Unde et hodie fratres nostri Praedicatores et Minores, qui predicaturi gentibus infidelibus per mundum dispersi sunt, maxime apud Tartaros multum frequenter faciunt fructum suum. Et frequenter ab eis audivi, quod hii qui per Dei gratiam convertuntur ad fidem valde constantes sunt et in fide valde devoti.» ${ }^{19}$

È quindi certo che Giovanni Colonna O. P., che s'interessava di materie orientalistiche, fu al corrente dell'attività dei Fratres Peregrinantes, o li conobbe di persona. Tra essi era probabilmente Collator, possessore di $P C A$, o qualche suo collaboratore (cf. infra § 3). È perciò possibile che Colonna conoscesse un lessico quale PCA o ne fosse pervenuto in possesso.

\section{Nomen}

L'attuale $P C A$ fu eseguito su carta tipo Bologna, esemplare conservato nell'Archivio di Stato della città (A. D. 1330-1331), che ha in filigrana il disegno di due pugnali incrociati (Briquet 5148). PCA è composto di due fascicoli. Il fascicolo I (ff. 1-13) manca dei tre fogli finali, che furono perduti con la messa in opera della legatura (c. XVIII secolo). Il fascicolo II appare completo (ff. 30-59). Tre poesie toscane, una ballata, una terzina e una quartina, furono apposte da mano di persona italiana in spazi finali già bianchi (f. $56^{\mathrm{v}}$ e $59^{\mathrm{r}}$ ). Queste poesie furono ritenute un «singolare cimelio di versi italiani che arieggiano la maniera di Petrarca». La nota di possesso apposta sul tomo II «Iste liber est de ant de Ziuale» (f. $78^{\mathrm{v}}$ ), persona ignota, fu letta Antonio de Ziuale o Zinale. ${ }^{20}$

${ }^{16}$ J. Richard: L'enseignement des langues orientales en Occident au Moyen Âge. Revue des Etudes Islamiques XLIV (1976), pp. 150-165.

${ }^{17}$ Cf. L. Manzoni: Di frate Francesco Pipini da Bologna de' pp. predicatori storico, geografo, viaggiatore del secolo XIV (1245-1320). Notizie inedite raccolte. Bologna 1896, pp. 43-73.

${ }_{18}$ A. D’Amato O. P.: I Domenicani e l'Università di Bologna. Bologna 1988, pp. 152-153.

${ }^{19}$ Iohannis de Columpna Mari Historiarum. In: Monumenta Germaniae Historica Scriptorum, ed. G. Waiz, XXIV. Hannoverae 1879, pp. 266-284: § 136.

${ }^{20}$ C. Frati: Versi italiani nel codice "Cumanico" della Marciana e F. Petrarca. Il Libro e la Stampa n. s. IV (1910), pp. 3-9. 
Il luogo di esecuzione di $P C A$, Solghat, e il tempo, c. 1330-1340, furono discussi da D. Rasovskij (Praga, 1929). Egli ribadiva la convizione per cui il "Codex Cumanicus" sia da attribuirsi a "Missionari francescani o domenicani, e a preferenza francescani» che operavano in Crimea. ${ }^{21}$ Il materiale linguistico di base sembra raccolto dal 1294-1295, e ordinato il «MCCCIII die $\mathrm{Xj}^{\circ} \mathrm{July»,} \mathrm{data} \mathrm{che} \mathrm{è} \mathrm{l'incipit} \mathrm{del}$ manoscritto. Un fascicolo III (ff. 60-82), unito ai due di $P C A$ in epoca incerta, è il frasario turco cumano-tedesco di genere missionario cristiano e di fattura germanica (c. 1340-1356). Il "Codex Cumanicus", tomo I (fascicoli I-II) e tomo II (fascicolo III), o anche soltanto PCA (tomo I), non appartenne a Petrarca, se fosse valido l'argomento che egli non collezionava libri cartacei, bensì esclusivamente membranacei. ${ }^{22}$

C. Desimoni, linguista ligure, lesse (1881) l'ex libris Antonio de Finale, che egli ritenne un ignoto mercante corregionale del XIV secolo. La data 1303 d'incipit fu considerata scrittura corsiva ligure, e così fu letta 1330, per consulenza di P. Zorzanello, direttore della Biblioteca Marciana di Venezia (1947). PCA sarebbe stato elaborato nel $1324-1325$ e quindi fissato nella copia che si conserva in Venezia. ${ }^{23} \mathrm{Il}$ lessico trilingue «was used by both Genoans and Venetians», mercanti o interpreti. «The existing copy was made in a Franciscan monastery around 1330 [... but it] was not suitable for missionary objectives [...] Strangely enough, the name of St. John does not occur at any other place in the codex. $\rangle^{24}$

Ma il nome di S. Giovanni Evangelista occorre in un altro codice, che è conservato nella Biblioteca Apostolica Vaticana e ho studiato di recente. Questo codice è Borg. pers. 19, "Quattro Vangeli" in traduzione persiana (A. D. 1338). Per collazione, correzione e integrazione del testo persiano, questo codice fu glossato da due coevi missionari latini. ${ }^{25}$ Chiamo il glossatore principale Collator. Tramite la qualità delle glosse, egli si dimostra un grande filologo e un perfetto conoscitore della lingua persiana scritta e parlata. Ritengo Collator probabilmente Giovanni da Firenze O. P., vescovo di Tiflis (1330-†Pera 1347), o un suo confratello. Essi operavano nella congregazione domenicana dei Fratres Peregrinantes pro Christo in Perside. Questa congregazione agiva in rapporto all'episcopato latino di Ordine Domenicano in Persia, Ecclesia Soltaniensis (Soltaniya, 1318-1425) e contigua Ecclesia Taurisiensis (Tabriz, 1329-1405), prima che la peste dell'anno 1348 ne provocasse una rovinosa falcidie. $^{26}$

${ }^{21}$ G. Golubovich O. F. M.: Bibliotheca bio-bibliografica della Terra Santa e dell'Oriente Francescano, III. Quaracchi 1919, pp. 1-28.

${ }^{22}$ G. Györffy: Autour du Codex Cumanicus. In: Analecta orientalia memoriae Alexandri Csoma de Körös dicata, I. Budapestini 1942, pp. 10-137.

${ }^{23}$ V. Drimba: Sur la datation de la première partie du Codex Cumanicus. Oriens 27-28 (1981), pp. 388-344.

${ }^{24}$ L. Ligeti: Prolegomena to the Codex Cumanicus. Acta Orientalia Academiae Scientiarum Hungaricae XXXV (1981), pp. 1-54.

${ }^{25}$ L'esistenza di glosse non fu segnalata in E. Rossi: Elenco dei manoscritti persiani della Biblioteca Vaticana. Città del Vaticano 1948, pp. 173-174, e P. Orsatti: Il fondo Borgia della Biblioteca Vaticana e gli studi orientali a Roma tre Sette e Ottocento. Città del Vaticano 1996, pp. 170, 173. Mia nuova descrizione del codice in Catalogo della mostra della Biblioteca Apostolica Vaticana I Vangeli dei Popoli, a cura bi F. D'Aiuto et al.: Città del Vaticano-Roma 2000, pp. 338-339.

${ }^{26}$ G. Fedalto: La Chiesa Latina in Oriente, I. Verona 1981, pp. 568-589. 
Il tratteggio dei caratteri, tra cui $\wedge$ notante la vocale a, in minuscola cancelleresca corsiva, distingue la mano di Collator. Le glosse concernono particolarmente il testo persiano del Vangelo di Giovanni. La terminologia delle glosse si mostra correlata a numerosi lemmi latino-persiani di $P C A$ (cf. $\S 6$ ). Nel frattempo Collator costituiva un lessico bilingue, o si serviva di uno come $P C A$, che era da lui posseduto. Al confronto dei tratti grafici, compreso il carattere $\wedge$ per a, la mano di Collator appare la stessa che appose le tre poesie toscane su $P C A .^{27}$

Inoltre «Iste liber est de ant de Ziuale» può leggersi ex-libris Antonio de Cividale, cioè «Antonius de Civitato (de Civitate Austriae, de Cividal) Conventus reformati S. Dominici de Venetiis alumnus (a. 1392)», allora sotto la giurisdizione di Giovanni Dominici O. P. Egli, Giovanni di Domenico Bianchini (Firenze 1357-Buda 1419), fondò il convento domenicano di Fiesole (1406). Allora Antonio da Cividale O. P. sembra avere qui seguito, almeno visitato Giovanni Dominici. ${ }^{28}$ Egli, cardinale, recò con sé al Concilio di Costanza (1415), come interprete, Beltramo Mignanelli (Siena 1370-1455), che allora scrisse una eccellente biografia di Thomorlengh, Tamerlano, per Sigismundus re di Ungheria. ${ }^{29}$ Beltramo certamente aveva incontrato Ioannes III O. P., vescovo di Nakhicevan, poi arcivescovo di Soltaniya. Ioannnes III O. P. fu autore di una bella cosmografia tascabile (c. 1410), e prima (1403) di una buona biografia di Themurlank. ${ }^{30}$

\section{Congregatio}

Dal 1289 erano in Tabriz due conventi francescani e uno domenicano. In Maragha l'opera dei Domenicani fu avviata almeno dal 1304. In riferimento a Soltaniya, la nuova capitale dei Mongoli di Persia, con la bolla Redemptor noster (1.IV.1318) papa Giovanni XXII (1316-1334), Jacques Dueze, ridistribuì l'episcopato missionario latino e consolidò la Società Domenicana dei Fratres Peregrinantes pro Christo. Ne partivano «50 missionarios novos pro partibus orientalibus» (1328); «eodem tempore in civitatibus Pera et Kaffa cathedrae pro linguis orientalibus erectae», e in «Armenia Maiori tunc temporis fr. Bartholomeus O. P. episcopus Maragensis». ${ }^{31}$

\footnotetext{
${ }^{27}$ Cf. Codex Cumanicus. Cod. Marc. Lat. DXLIX in Faksimile herausgegeben mit einer Einleitung von K. Grønbek. Kopenhagen 1936, ai fogli rispettivi.

${ }^{28}$ Th. Kaeppeli O. P.: Scriptores Ordinis Praedicatorum in Medii Aevi. Romae, I, 1970, pp. $110-111$.

${ }^{29}$ A. M. Piemontese: La lingua araba comparata da Beltramo Mignanelli (Siena 1443). Acta Orientalia Academiae Scientiarum Hungaricae XVLIII (1995), pp. 155-170. Id.: Beltramo Mignanelli senese biografo di Tamerlano. Oriente Moderno LXXVI (1996), pp. 213-226.

${ }^{30}$ A. Kern: Der "Libellus de Notitia Orbis" Ioannes III. (De Galafontibus?) O. P. Erzbischofs von Sulthaniyeh'. Archivum Fratrum Praedicatorum VIII (1938), pp. 82-123; C. Delacroix-Besnier: Les Missions Dominicaines et les Arméniens du milieu du XIV ${ }^{\mathrm{e}}$ siècle aux premières années du XV $\mathrm{XV}^{\mathrm{e}}$ siècle. Revue des Études Arméniennes 26 (1996-97), pp. 173-191.

${ }^{31}$ B. M. Reichert: Acta Capitulorum Generalium Ordinis Praedicatorum, II. Romae 1899, p. 176; M. A. Van den Oudenrijn O. P.: Historia generalis brevissima missionum ordinis Fratrum Praedicatorum. Analecta sacri ordinis Fratrum Praedicatorum a. XXVII, vol. XIV (1919), 
Egli, Bartolomeo da Poggio, detto da Bologna, che forse procurò le risme della carta per $P C A$, fu il primo vescovo latino di Maragha (1328-†Kirnë 1333). Secondo e ultimo vescovo fu allora Gui da Cortona O. P. († c. 1347). In Diacorogon, ossia Dihxwāragān, villaggio strategico presso Maragha, furono vescovi Gérard Calvet de Monpellier O. P. (1318-1322) e Bernardo di Guardiola O. P., forse spagnolo (1329-1349). In Tabriz furono vescovi latini Bartolomeo Abagliati da Siena (c. 1318-1329), Guglielmo de Cigiis O. P. (c. 1333), Francesco Cinquini da Pisa O. P. († Pisa 1348). Cinquini dal 1333 fu vicario generale dei Fratres Peregrinantes pro Christo in Perside. In Soltaniya, dal 1321 fu attiva una casa domenicana, con un arcivescovo latino. Il terzo arcivescovo era Giovanni da Cori O. P. (1329-1333 almeno). Il quarto arcivescovo, residente ante 9.IX.1343, fu Guglielmo de Cigiis O. P. († 1348), già vescovo di Tabriz (1329).

Francesco Cinquini e Gui da Cortona, inoltre Bernardo di Guardiola, possono entrare nella lista delle persone idonee per identificare Collator. Ma si arguisce che Collator debba avere attinenza con l'attività compiuta da Bartolomeo da Poggio O. P., il teologo, filosofo, filologo che organizzò i Frati Unitores (Armeni reuniti). Egli animò la celebre officina di traduzione di testi latini in armeno, in Maragha (13281333), quindi (1330-1333) nel monastero superiore di Kirnë sull'Ernčak, persiano Alanjak. ${ }^{32}$

Per iniziativa di Yovhannes da Kirnë O. P. († 1348), il monastero della S. Madre di Dio (s. Astuacacin de K'rna), sorse (1330) e fu donato all'Ordine Domenicano (1331). Yakobos da Kirnë O. P. Tarjumān 'interpres' fu il coadiutore di Bartolomeo, e poi del suo successore. Nel comunicare e tradurre egli e Yakobos si servivano del persiano quale lingua veicolare comune. ${ }^{33}$ "Ils prêchaient en langue persane», come André de Longjumeau O. P. e i confratelli Domenicani peregrinanti in questi paesi dell'Asia. ${ }^{34}$

L'opera di Bartolomeo da Poggio fu continuata dal suo successore nel convento di Kirnë, Iohannes de Florentia O. P., episcopus Tepheliciensis. Egli dal 1333 diresse la missione domenicana d'Armenia e la congregazione dei Frati Unitori di Kirnë, che adottarono la liturgia latina dinanzi a lui (1337). Iohannes de Florentia «tria varia ydiomate perfectissime didicit in quibus diversis orientalum nationum populis xpm summa verbi efficacia predicavit», riferisce il suo necrologio in Santa Maria Novella di Firenze. ${ }^{35}$ Una delle tre lingue era la georgiana, sembra; un'altra poteva essere la persiana, la terza la turca o l'armena. «En Orient il se signala par son aptitude pour l'étude des langues, à tel point qu'on le fit ordonner prêtre [...] Au cour des années 1343/1345 nous le trouvons en Europe [...] Il est mort au couvent de Péra en

pp. 30-34; J. Richard: La Papauté et les missions d'Orient au Moyen Age (XIII $-X V^{e}$ siècles). Rome 1977, pp. 170-225.

${ }^{32}$ M. A. Van den Oudenrijn: L'évêque dominicain Fr. Barthélemy fondateur supposé d'un couvent dans le Tigré au $14^{\mathrm{e}}$ siècle. Rassegna di Studi Etiopici V (1946), pp. 7-16. 186-207.

${ }^{33}$ Id.: Linguae Hicanae Scriptores. Bernae 1960, p. 20; inoltre pp. 26-28, 128, 173,

${ }^{34}$ Id.: Uniteurs et Dominicains d'Arménie. I. Oriens Christianus 40 (1956), pp. 94-112.

${ }^{35}$ Id.: Annotationes Bibliographicae Armeno-Domenicanae, II. Analecta Sacri Ordinis Fratrum Praedicatorum XVIII (1920), pp. 280-294. 
1347 et fut enceveli dans l'église du couvent Saint-Dominique.» La sua lastra funeraria, inscritta Ioannes de Florentia, fu trasportata nel Museo Topkapı d'Istanbul. ${ }^{36}$

Giovanni da Firenze O. P., vescovo di Tiflis, parrebbe assai vicino a Xwāja Amīr ibn Sahm al-Dawla ibn Širāna, «noto come Tiflizī»), un certo signore di Tiflis per la cui committenza il copista Šim'ūn ibn Yūsuf ibn Ibrāhīm al-Tabrīzī eseguì in Caffa (1341) un vangelo persiano tradotto dal siriaco (Bodleian 1835), poi edito. ${ }^{37}$ È il quarto testimone conservato del genere, cioè in ordine cronologico successivo al Borg. pers. 19.

\section{Emendatio}

Il codice Borg pers. 19 "Quattro Vangeli", datato A. D. 1338, cartaceo, mm 160×120, legatura armena del XVII secolo, abbina due tomi modesti di diversa fattura: I Matteo (ff. $1^{\mathrm{r}}-48^{\mathrm{v}}$ ) e Marco (ff. $50^{\mathrm{v}}-88^{\mathrm{v}}$ ), mutilo di almeno una guardia anteriore, gravemente frammentario e in disordine, inoltre testo viziato da gravi corruttele; II Luca (ff. $93^{\mathrm{v}}-177^{\mathrm{r}}$ ) e Giovanni (ff. $180^{\mathrm{v}}-243^{\mathrm{v}}$ ), mutivo di vari fogli e apodo, il cui copista si firma (f. $177^{r}$ ) Żiyā al-Dīn xwāja ibn al-ra'ūf mawlā nā'ib Nūr al-Dīn ma'rūf baKilamjī al-Ifranjīi, in data 15 ša'bān 738 [H.]/ A. D. 8.III.1338.

La firma del copista significa «Messer Lucentezza della Fede figlio del benigno maestro vice Luce della Fede, noto come l'Oratore Franco.» Oratore Franco pare calco o sinonimo persiano di 'Predicatore Latino' (ifranjī, farang $\bar{l}$ ), se egli era nell'ordine dei Domenicani; ovvero di 'Missionario Europeo', anche in caso egli fosse francescano. Il termine kilamčč forma mongola originale kilamači, 'oratore', è attestato come nunzio-interprete, messo straniero di lingua e fede, nella celebre storia enciclopedica universale che raccolse (1304-1316) il medico ebreo e ministro Rašīd aldīn (Hamadan c. 1247-Tabriz 1317) per i regnanti mongoli di Persia. ${ }^{38}$

I due tomi di Borg pers. 19, eseguiti per missionari latini, furono uniti o riordinati da Collator. Egli appose a inchiostro nero: I) in ogni foglio, testatine identificative di ciascun capitolo e paragrafo dei singoli vangeli, in base alla divisione di rito latino; II) a margine le didascalie inerenti i passi laddove occorra leggere e recitare conforme la liturgia romana; III) la relativa lista delle feste e ricorrenze (ff. $49^{\mathrm{r}}-50^{\mathrm{r}}$ ), talora tracciando A invece di $\wedge$; IV) sovente la sigla $«$ F», scil. Feria.

Inoltre, in scrittura minuscola cancelleresca molto corsiva, Collator segna con acribia filologica glosse interlineari, marginali e in calce. Le glosse concernono termini di pericopi di Matteo (1 glossa leggibile) e di Luca (37 glosse), inoltre termini (29 glosse) e pericopi (25 glosse) di Giovanni. Una seconda persona, che chiamo $\mathrm{Se}$ quior, appose qualche glossa sporadica, di scarsa qualità filologica.

${ }^{36}$ R. Loenertz O. P.: La société des Frères Pérégrinants. Romae 1937, pp. 173-174.

${ }^{37}$ E. Sachau et al.: Catalogue of the Persian [...] Manuscripts in the Bodleian Library, I. Oxford 1899, pp. 1053-54; Brian Walton (ed.): Biblia polyglotta, V. Londini 1657, pp. 5-515.

${ }^{38}$ Rašīd al-Dīn F. Hamadān̄i, Jāmi' al-tavārēxx, ed. M. Roušan. Tehrān 1373/1994, I, p. 687; III, p. 1263; IV, p. 2400. 
Per collazionare ed emendare il testo persiano, Collator ne traduce e traslittera singoli termini in scrittura latina diacritica. Inoltre egli traslittera pericopi intere. Collator teneva presenti almeno due antigrafi persiani diversi, redatti in scrittura araba. Essi recavano un vangelo completo, caratterizzato da lezioni arcaiche, e un vangelo di Giovanni tradotto dal latino. In sostanza la glossatura verte sul Vangelo di Giovanni, al fine di ottenerne una traduzione persiana corretta in rapporto al testo base latino.

Collator applica un sistema di traslitterazione che si fonda sull'osservazione scientifica della scrittura arabopersiana. Il suo caratteristico metodo scrittorio della punctatio fu compreso da chi inventò tale traslitterazione. Essa anticipa in buona parte quella di uso persianistico e arabistico moderno. In particolare, un punto diacritico è scritto a fianco destro di queste lettere, a mezza altezza: $\mathbf{g} \cdot\langle\mathrm{g}>, \mathbf{k} \cdot<\mathrm{x}>, \boldsymbol{i} \cdot($ iḍa $f a)$, per lo $i$ più scritta senza il punto a fianco e in forma abbreviativa latina. Con $\dot{\mathbf{g}}$ un punto diacritico è scritto sopra la lettera $g$ per notare $<\dot{\mathrm{g}}>$, lettera $\dot{g} a y n$. Con å un punto è scritto sopra la lettera $a$ per notare $\left.<^{6}\right\rangle$, lettera 'ayn: preceduta o seguita dal fonema $a$, che tipograficamente rendo così å. Tre punti, tal quale l'araba šīn, sormontano la lettrea $s$, che tipograficamente rendo š. La copula est $<a s t>$ è per lo più scritta in una forma siglata a zigzag tipica della latina est. ${ }^{39}$

\section{Collatio}

Si confrontano collimanze e, in più, forme corrette che vari termini delle glosse latine e latinopersiane su Borg. pers. 19 prefigurano rispetto a numerosi lemmi di PCA. Vi si può, sia correggere, cosa già compiuta dai filologi cumanisti, sia spiegare meglio la presenza di certi lemmi, in $P C A$. Cito qui gli esempi più significativi. Segno in neretto i termini delle glosse apposte da Collator, in corsivo i termini del testo persiano, i lemmi di $P C A$ tra virgolette basse «", e la traduzione-edizione critica latina dei Vangeli a cura di A. Merk S. J. (1948) tra virgolette alte “".

lacrimas traduce $a s ̌ k h \bar{a}$ (f. $\left.118^{\mathrm{r}}, 1.1\right)$. L 7,38. PCA «lacrima asch». zingil»».

zanjír catena traslittera e traduce zanjīr (f. 129v, 1. 4). L 8,29. PCA «catena

sengui grauis traslittera e traduce sangī da pas Šam 'ūn dar $x w \bar{a} b-i$ sangī šud (f. 132 v, 1.3 ). L 9,32 "Petrus vero et qui cum illo erant gravati erant somno". PCA «grauis sanguj».

reliqui traduce il primo termine di bāqū-i Jalīliyān (f. $\left.125^{\mathrm{v}}, 1.8\right)$. L 13,3. PCA «remanens batchi».

La glossa di Collator riguardante Luca 9,32 è un termine chiave. Esso può giustificare l'inserzione del lemma analogo in PCA. Termine chiave, come forma rara, un hapax che ancora non registrano i dizionari della lingua persiana di autori autoctoni, è il paradigma del lessico «rogo lauagâ̂ mecunê», «rogauj lauagaî cher-

${ }^{39}$ Cf. B. Bischoff: Paläographie des römischen Altertums und des abendländischen Mittelalters. Berlin 1979, p. 222, tipo centrale ivi descritto in $§ 7$. 
dem», «rogamen lauagâे». ${ }^{40}$ Per la correzione di questo paradigma serve la buona lezione lauagarí kaerdénd nelle pericopi seguenti.

$i$ an zán ki guaha medad oa meg·uft ki ba mén gufta est hár chi kerdá am. aknun chon samarían peš $i$ åysa amadénd lauagarí kaerdénd oura ta anja muḳam g•irád. oa du roz anja mand. oa ådian psiartar ${ }^{41}$ bauar kerdénd az baray sok•on $i$ ou. oa meguftánd ba an zán. ki In saât ná tonha az baray kalíma i ou bauar míarím. zeraki ma k•od šínidím oa danedim ki ba haḳikát In ast k•alaskunanda $i^{42}$ duniâ (f. $189^{\mathrm{r}}$ e $188^{\mathrm{v}}$, a fronte, in calce) emenda e integra una lacuna del testo persiano, per salto del copista (f. 189r , 1. 13). J 4, 39-42 “(Ex civitate autem illa multi crediderunt in eum Samaritanorum propter verbum) mulieris testimonium perhibens: Quia dixit mihi omnia quaecumque feci. Cum venissent ergo ad illum Samaritani, rogaverunt eum ut ibi maneret. Et mansit ibi duos dies. Et multo plures crediderunt in eum propter sermonem eius. Et mulieri dicebant: quia non propter tuam loquelam credimus; ipsi enim audivimus et scimus quia hic est vere Salvator mundi”. $P C A$ «illi ba an», nominativo an, «quam chi», «testifico gua medaem» («testis gua»: guah), «dissi guften» («dico megoen»), «michi baman», «omne ghar»: har, «quid či $\rangle$, «factum cherda», «ergo acum»: acnun, «quomodo chion», «aput [apud] pes», «venj anmadem», «rogauj lauagaî cherdem», «suum oura», «donec ta», «illuc ania», «dies rox», «mansi mandem» («maneo memaanem»), «et ou», «nimis pisiar» («satis bisiar», «multum bisiar»), «credidi bauar cherdem» («credo bauar mecunem»), «ab az», «propter barayn», «fides bauar», «aporto miauarem», «nunc inscaat»: insaat, «non na», «solus tanaha», «quia cera», «nos yma», «audivimus sinidim», «sciuj danisten» («scio medanem»), «ille, iste in, uel ou», «est est», «redimo chalas mecunem» («redemptio chalasi»), «mondus [mundus] dunia».

agar kase murad $\boldsymbol{i}$ au ba jay aurdén bšinaséd az âmuziš $\boldsymbol{i}$ men agar az k•oda ast emenda e varia (f. 199v, 1. 12) har ki xwāhad ki ba murād-i $\bar{u} k \bar{a} r$ kunad gū 'ilm-i man dar yāb ki az xud̄a yā tān- $\bar{l}$. J 7,17 "Si quis voluerit voluntatem eius facere, cognoscet de doctrina, utrum ex Deo sit”. PCA «si agar», «persona chas», «aportauj auurdem», «cognosco mesnasem», «disce biamuz», «ego man», «deus ghoda».

bar mén k·ašm mag·ired emenda e varia bar man čirā mī dndīd bsīt [! ] (f. $\left.200^{\mathrm{r}}, 1.13\right)$. Corruttela grave delle due ultime parole nel testo persiano; la seconda parola sta per la forma arcaica $b \bar{a} s \bar{t} t$ 'siate'. J 7,23 “mihi indignamini". $P C A$ «capio megiren».

adamíra támam (f. $200^{\mathrm{v}}, 1.1$, margine destro) integra il complemento oggetto mancante nella frase (f. 200r, 1.13 ) dar rūz-i šanba (f. $200^{\mathrm{v}}, 1$. 1) šifā dādam. J 7,23

\footnotetext{
${ }^{40}$ Lemma diverso «grauis sangun» per <sangīn>, cf. Codex Cumanicus Bibliothecae ad Templum divi Marci Venetiarum, ed. Géza Kuun. Budapestini 1880, pp. 52, 76, 87, 331, 335; D. Monchi-Zadeh: Das Persische in Codex Cumanicus. Uppsala 1969, pp. 104, 141; A. Bodrogligeti: The Persian Vocabulary of the Codex Cumanicus. Budapest 1971, pp. 160-161, 189. Cf. anche K. Grønbek: Komanisches Wörterbuch. Türkischer Wortindex zu Codex Cumanicus. København 1942.

${ }^{41}$ Forma comparativa $<$ bisyārtar $>$ di $<$ bisyār $>$ 'molto'; il copista del testo persiano scrive

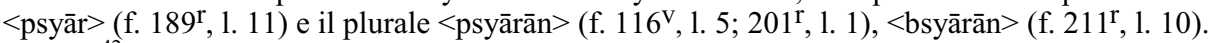

${ }^{42}$ Qui l'iḍa $f a$ è trascritta $i$ con punto a fianco.
} 
"(Quia) totum hominem (sanum feci in sabbato?)". PCA «compleo tamam mecunê», «complementum tamam».

šariåt $\boldsymbol{i}$ rujatí makuned, cioè "non fate giudizio opinabile", anche "visivo, de visu' <ru'yatī>, emenda mabāš̄ $\underline{d}$ ḥākimān ba-nifāq (f. 200v, 1. 1, margine sinistro). J 7,24 "Nolite iudicare secundum faciem". PCA «judico», «judicaui», «sentencia», «sententia»: lemmi bianchi, sprovvisti dei corrispettivi termini persiani.

saruaran ba au hiz ná me g•oyénd emenda bā $\bar{u}$ namītavānīd guftan magar pīrān (f. $200^{\mathrm{v}}, 1.4$ ). J 7,26 "et nihil ei dicunt. Numquid vere cognoverunt principes". Il termine $<$ hīz $>$ hiz è attestato in persiano arcaico. ${ }^{43}$

oa agar bgoyém ki oura ná mešnasém. mešl $\boldsymbol{i}$ šmâ drog் go bašém [man cassato] oura mešnasém va medaném varia il testo persiano (f. 200v). J 7,29 "et si dixero quia nescio eum ero similis vobis mendax et scio eum”. Questa variante è inserita, per anticipo di $\mathrm{J} 8.55$, in antichi manoscritti latini. ${ }^{44} P C A$ «mendax drogoan», «false badrog».

šma šaríat $\boldsymbol{i}$ sauratí < tauratī> me kuned. - šma bar har gušt mâdi šariåt mekuned emenda con variante duplice šuma rā hukm hast jismānī (f. $203^{\mathrm{v}}, 1$. 5). Il testo persiano collima con la lezione siriaca, "vos corpolariter judicatis". 45 J 8,15 "vos secundum carnem iudicatis". PCA «vos ysma» («vobis ba suma», accusativo «vos sumara»), «caro goist», «brionus ghost i baxou» [gốst-i bāzu], «femina maya».

oa k•y dašt o ân chi frstada mešud meburd ba k•od emenda barāy-i anki

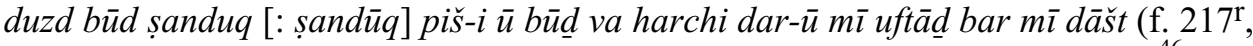
1. 11-12). Anche il termine $<x \overline{\mathbf{1}}>\mathbf{k} \cdot \mathbf{y}$, cioè 'otre', è attestato in persiano arcaico. ${ }^{46}$ Forma comune di $\langle x \overline{\mathbf{1}}\rangle$ è $<\mathrm{x} \overline{\mathrm{k}}\rangle$. J 12,6 "et loculos habens ea quae mittebatur portabat". $P C A$ «habuj dastem», «missus frista», «factus sum sudem» («fio mesauem»), «portauj burden» («porto mebarem»).

ta dar roz ba g•or kerdán $\boldsymbol{i}$ mán. anra níg•ah daréd emenda guft ba $\bar{u} \bar{I} s \bar{u}$ ' bigud̄ār $\bar{u} r \bar{a}$ ki rāz [: az] rūz-i dafn karrdna [: kardan] nigāh dāšta ast (f. $217^{\mathrm{r}}$, in calce). J 12,7 "Dixit ergo Jesus: Sinite illum ut in diem sepulturae meae servet illud". $P C A$ «donec ta», «in dar», «dies rox», «subterrauj dar ghor neadem», «custodia nigaa»; «habeo medarem»;

meayéd emenda la forma del verbo in inak malik-i to amada (f. 218 r , 1. 1). J 12,15 “ecce rex tuus venit". PCA «miayat», «venio miaen».

oa inra b vay kerdénd integra la frase omessa nel testo persiano (f. $218^{r}$, 1. 4). J 12,16 "et haec fecerunt ei".

ki ma hech sud na mekunim zera ki inak integra la frase omessa nel testo persiano (f. $218^{\mathrm{r}}, 1.9$ ). J 12,19 "Videte quia nihil proficimus? ecce". PCA «nichil heč», «ecce anecha».

${ }^{43}$ A. B. Asadī Țūsī, Luġat-i Furs, ed. 'A. Iqbāl. Tehran 1319/1940, p. 172.

${ }^{44}$ Cf. Novum Testamentum Graece et Latine, ed. A. Merk S. J. Romae 1948, p. 335.

${ }^{45}$ Cf. Tretraeuangelium Sanctum, iuxta simplicem syrorem versionem, ed. Ph.-E. PerseyG. H. Gwilliam. Oxonii 1901, p. 529.

${ }^{46}$ Asadī Țūsī: op. cit., p. 520. 
harke šak $\cdot \mathbf{s}^{47}$ i k•odra dost dared oura gum kunéd. oa harki šak·s $\boldsymbol{i}$ k•odra dar in jehan dušman daréd. ba zendganí $\boldsymbol{i}$ javid oura nigah daréd emenda har $k i$ jān-i xwod̂drā dušman [lapsus di dust, abraso e riscritto] dāšt dar-īn jihānn nigāh-aš $d \bar{a} s ̌ t$ (f. 218v). J 12,25 "Qui amat animam suam, perdet eam; et qui odit animam suam in hoc mundo, in vitam aeternam custodit eam". PCA «omne ghar»: har, «amat dost medaret», «vita xendaganj», «custodio nigaa mecunem», «custodia nigaa»: nigāh.

hanuz dar šma nur ham ast emenda andak zamān-i dīgar nūr bā šumā bāšad (f. 219v, 1. 1»). J 12,35 "Adhuc modicum lumen in vobis est". PCA «adhuc ohanux» («chanux»), «lux nur»;

katan traslittera katān (f. $\left.238^{\mathrm{v}}, 1.12\right)$. J 20,5. PCA «tella katan, chetan».

dastarcha <dastārča $>$ varia 'imāma (f. 239r , 1. 1). J 20,7. PCA «toalia dastar».

Come si vede, per quanto riguarda $P C A$, ossia la parte latino-persiana del " $\mathrm{Co}$ dex Cumanicus", questo illustre cimelio non è più un testimone unicum né isolato. Altri studiosi potranno compiere un confronto analitico fra la terminologia delle glosse su Borg. pers. 19 e i lemmi di PCA. Ciò concerne anche un commento delle particolarità grammaticali e fonologiche della lingua persiana testimoniate da Collator.

Pubblico altrove l'edizione integrale delle glosse e un'analisi del sistema di traslitterazione scientifica che esse documentano. ${ }^{4}$

Con la testimonianza paleografica dell'opera filologica di Collator, si può ora ridisegnare la storia della conoscenza europea della lingua persiana durante l'età medioevale. Così si rintraccia anche l'iter di trasmissione della celebre Tārīx-i Farang dal latino in persiano. ${ }^{49}$

${ }^{47}$ Arabo šaxṣ: significa anche «figure» e «rapprésentation d'une personne absente», cf. R. Dozy: Supplément aux Dictionnaires arabes. Leyde 1881, I, p. 734.

${ }^{48}$ Cf. A. M. Piemontese: Le glosse sul Vangelo persiano del 1338 e il Codex Cumanicus. Miscellanea Bibliothecae Vaticanae VIII (2000), in corso di stampa.

${ }^{49}$ Id.: La via Domenicana verso la Cronaca Europea di Raš̄ìd al-Dīn. In: Miscellanea in memoria di Aldo Gallotta. Napoli, Istituto Universitario Orientale, 2000, in corso di stampa. 